Method Between August 2011 and June 2014, 235 patients underwent MVP, of which $71(30.2 \%)$ had a MI procedure performed through a 5-6 $\mathrm{cm}$ right anterior minithoracotomy. We adapted the Composite Physical Function questionnaire to retrospectively assess post-operative quality of life in 3 domains (scores were converted into a 0-100 scale) -- Recovery Time (higher score indicated longer recovery time); Pain (higher score indicated increased pain) and Treatment Satisfaction (higher score indicated improved satisfaction). The scores were risk-adjusted using pre-operative characteristics (age, gender, urgency, Logistic EuroSCORE). Data are reported as median (interquartile range).

Results The response rate was 70.6\% ( $\mathrm{n}=166)$ of which 51 (30.7\%) underwent MI repair. Comparing St to MI, $14.8 \%$ vs $35.3 \%$ resumed 'normal activities' within 6 weeks of their operation $(\mathrm{p}=0.003)$. The risk-adjusted Recovery Time domain results for St vs MI were 54.4 (44.4,69.6) vs. 44.9 (26.7, 58.0), $\mathrm{p}<0.001$; likewise, the risk-adjusted Pain domain results were $31.8(16.9,50.0)$ vs. 21.9 (12.4, 32.8), p $=0.03$; and the risk-adjusted Treatment Satisfaction domain results were $98.1(83.9,99.9)$ vs. $98.3(88.9,100.0), \mathrm{p}=$ 0.25 . Comparing St to $\mathrm{MI}, 53.0 \%$ vs. $78.4 \%$ reported that they were 'Very Satisfied' with the appearance of their scar ( $p$ $=0.002$ ).

Conclusion Minimally invasive mitral valve repair speeds recovery, reduces pain and improves cosmesis compared to a sternotomy approach. Both approaches score highly on patient satisfaction.

\section{PREVALENCE OF AORTIC VALVE ABNORMALITIES IN THE ELDERLY: A SCREENING STUDY USING HAND-HELD ULTRASOUND SCANNI}

${ }^{1}$ Catrin Williams*, ${ }^{2}$ Nicholas Maskell, ${ }^{3}$ Emma Rees, ${ }^{4}$ Kirstie Truman, ${ }^{2}$ Adrian lonescu. ${ }^{1}$ Nywel Dda NHS Trust; ${ }^{2}$ Morriston Hospital; ${ }^{3}$ Swansea University; ${ }^{4}$ Bay Area Practice; *Presenting Author

\subsection{6/heartjnl-2016-309890.157}

Background Degenerative aortic stenosis (AS) is increasingly recognised and treated with valve replacement in the elderly. The advent of percutaneous aortic valve intervention (TAVI) has opened opportunities for treatment in patients who would have been turned down for surgery just a few years ago, with a commensurate increase in health care-associated costs. However, there is a relative lack of data regarding the prevalence of AS in this very group of older patients, which can make planning and delivery of a TAVI service difficult.

Aim of the study

We set out to investigate the prevalence of aortic valve abnormalities by performing echocardiography with a handheld scanner in elderly patients attending a large GP surgery for non-cardiac reasons. We hypothesised that a strategy of echo screening for AV abnormalities in this population would have a high yield in detecting clinically relevant pathology.

Methods One hundred consecutive patients aged $>75$ years old who had no history of a heart murmur, myocardial infarction or heart failure had a detailed echocardiographic exam using a hand-held scanner without spectral Doppler capabilities. Cardiac risk factors were documented. We classified aortic valves as normal, aortic sclerosis (thickened leaflets without restriction) and aortic stenosis (thickened, restricted leaflets). Qualitative LV function assessment and colour Doppler assessment of aortic and mitral regurgitation were also obtained.
Results The mean age was 78.3 (8.7) years. The quality of the images was good in 74 studies, moderate in 20 and poor in 6. We found 48 normal valves, 28 cases of aortic sclerosis and 24 of aortic stenosis (of which 4 moderate and 1 severe). Aortic regurgitation was present in 35 cases $(30$ mild, 5 moderate), and LVEF was normal in 92 subjects, mildly impaired in 6 and moderately impaired in 2. There were 2 cases of severe MR and 4 of moderate MR, all organic. Neither cardiac risk factors nor mitral annular calcification were associated with aortic sclerosis or stenosis.

Conclusions Our preliminary data suggest that a strategy of echocardiographic screening in patients $>75$ years of age identifies a significant number of abnormalities that require formal cardiology follow-up or work up for intervention, including a $5 \%$ prevalence of moderate or severe AS, and $6 \%$ prevalence of moderate or severe MR.

\section{NT-PRO BNP AND SURVIVAL IN AORTIC STENOSIS}

${ }^{1}$ Tamir Malley*, 'Vassilis Vassiliou, 'Dominique Auger, 'Claire Raphael, ${ }^{2}$ Helen Berry, ${ }^{1}$ Eva Nyktari, ${ }^{1}$ Francisco Alpendurada, ${ }^{2}$ Jackie Donovan, 'Dudley Pennell, 'Sanjay Prasad. ${ }^{1}$ Royal Brompton Hospital; ${ }^{2}$ Department of Clinical Biochemistry; * Presenting Author

\subsection{6/heartjnl-2016-309890.158}

Introduction Elevated NT-pro BNP is associated with worse outcome in patients with cardiomyopathy.

Hypothesis We hypothesized that a mild elevation in NT-pro BNP may be associated with a worse outcome in patients with Aortic Stenosis (AS).

Methods Between 2011 and 2015, consecutive consenting patients with moderate $\left(1.0-1.5 \mathrm{~cm}^{2}\right)$ or severe $\left(<1 \mathrm{~cm}^{2}\right)$ AS considered for aortic valve intervention (either for severe AS or moderate AS with coronary disease) who had a cardiovascular magnetic resonance (CMR) scan undertaken for anatomy and function and also had blood stored for biomarker analysis were included. NT-pro BNP was measured using one-step immunoassay sandwich method with a final fluorescent detection (ELFA). Survival was obtained from hospital notes, electronic records and the National Strategic Office.

Results 112 patients $(76 \pm 10$ years, 78 male) were included. The group was dichotomised according to NT-pro BNP value, into normal NT-pro BNP value group (values from 0-300 pg/ $\mathrm{ml}, 23$ patients) and high group (>300 pg/ml, 89 patients). End point was all cause mortality. At a median of 12 months follow-up, 21 patients had died, all from the high NT-pro BNP group. The high NT-pro BNP group had significantly

\section{Survival in Low and High NT- pro BNP groups}

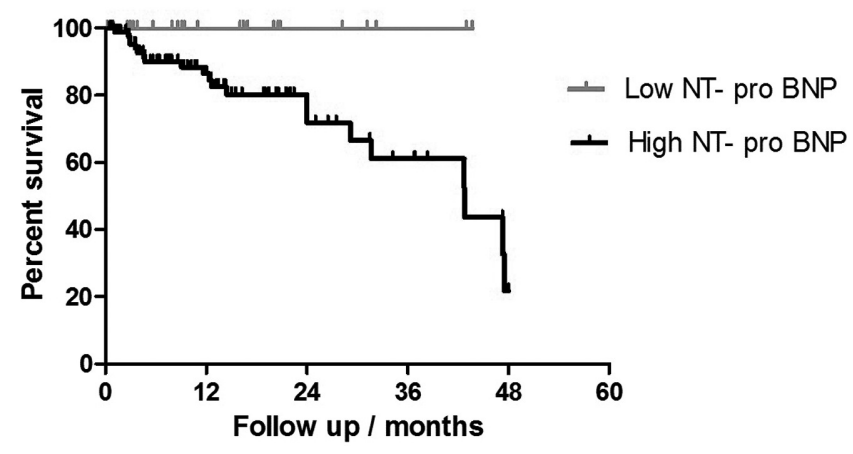

Abstract 158 Figure 1 Survival in low and high NT - Pro BNP groups 